\title{
Correction: HLA-C*07 in axial spondyloarthritis: data from the German Spondyloarthritis Inception Cohort and the Spondyloarthritis Caught Early cohort
}

Janneke J. de Winter ${ }^{1}$ - Iris C. Blijdorp ${ }^{1,2}$ • Henriëtte M. de Jong ${ }^{1}$ - Jürgen Sauter ${ }^{3}$ - Alexander H. Schmidt ${ }^{3}$. Floris A. van Gaalen ${ }^{4}$ - Désirée van der Heijde ${ }^{4}$. Denis Poddubnyy ${ }^{5} \cdot$ Nataliya G. Yeremenko $^{1,2}$. Marleen G. van de Sande ${ }^{1} \cdot$ Dominique L. Baeten ${ }^{1}$

Published online: 9 May 2019

๑) Springer Nature Limited 2019

\section{Correction to: Genes \& Immunity}

https://doi.org/10.1038/s41435-019-0061-4

published online 26 February 2019
The original version of this Article contained an error in the spelling of the author Denis Poddubnyy, which was incorrectly given as Denis Podubbnyy. This has now been corrected in both the PDF and HTML versions of the Article.

Dominique L. Baeten

d.1.baeten@amc.uva.nl

1 Department of Clinical Immunology and Rheumatology, Amsterdam Rheumatology and immunology Center, Academic Medical Center/University of Amsterdam, Amsterdam, The Netherlands

2 Laboratory of Experimental Immunology, Academic Medical Center/University of Amsterdam, Amsterdam, The Netherlands

3 DKMS German Bone Marrow Donor Center, Tübingen, Germany

4 Department of Rheumatology, Leiden University Medical Center, Leiden, The Netherlands

5 Department of Rheumatology, Charité Universitätsmedizin Berlin, Berlin, Germany 